\title{
High-resolution photoelectron spectrometry study of conjugate shakeup processes in the $\mathrm{Li} 1 \mathrm{~s}$ threshold region
}

\author{
B. Langer, J. Viefhaus, O. Hemmers, A. Menzel, and R. Wehlitz \\ Institut für Strahlungs-und Kernphysik, Technische Universität Berlin, D-1000 Berlin 12, Federal Republic of Germany \\ U. Becker \\ Fritz-Haber-Institut der Max-Planck-Gesellschaft, D-1000 Berlin 33, Federal Republic of Germany
}

(Received 7 September 1990)

\begin{abstract}
Partial cross sections and angular-distribution asymmetry parameters of diagram and satellite lines associated with $\mathrm{Li} 1 s$ photoionization were measured using synchrotron-radiation excitation. Special emphasis was given to a high-resolution study of the ${ }^{1} \boldsymbol{P}$ and ${ }^{3} \boldsymbol{P}$ conjugate shakeup satellite lines testing qualitative predictions of the conjugate shakeup model: increasing $\sigma$ and decreasing $\beta$ values towards threshold, both being verified. Comparison with recent relaxed Hartree-Fock calculations shows good agreement for the ${ }^{1} \mathrm{P}$ satellite, but also demonstrates that the present theory does not seem to be able to describe the cross-section behavior of the ${ }^{3} P$ satellite correctly.
\end{abstract}

The interest in atomic and molecular photoionization has moved continuously towards a deeper understanding of the role and importance of correlation effects in photoionization. $^{1,2}$ More specifically this has been accomplished in most cases by critical comparison between theoretical results, which include correlation effects, and improved and extended sets of experimental data. However, these effects can be more directly studied via multielectron processes such as photoelectron satellites and shakeoff electrons, particularly near threshold and on resonances. From a purely phenomenological point of view, satellites reflect the probability of the target system to remain after ionization in an excited ionic state rather than in the ionic ground state or, more generally, in a one-hole final state as expected on the basis of the oneelectron model. The study of the energy dependent behavior of such satellite lines in photoelectron and Auger spectra brings out specific information about the underlying electron correlations. ${ }^{3}$ The $\mathrm{He}^{+}(n=2)$ satellite behavior was one of the first examples where the controversy between the results of two different theoretical approaches, ${ }^{4-6}$ was conclusively resolved on the basis of the near-threshold behavior, in this case of the angulardistribution asymmetry parameter $\beta .^{7,8}$

In a similar sense to helium being the simplest closedshell atom, lithium is the simplest open-shell atomic system besides hydrogen. This makes $\mathrm{Li}$ an excellent choice to study, particularly because the effects of electron correlation on the photoionization process can be quite large due to this simplicity: One third of the electrons leave the atom. The principal core photoionization processes in atomic Li split into three different classes, the main line or diagram photoionization, the "shakeup" transitions and the "conjugate shakeup" transitions, the latter representing a process in which the valence electron exchanges angular momentum in addition to energy with the photoelec- tron. These processes may be written as follows:

$$
\begin{aligned}
1 s^{2} 2 s\left({ }^{2} S\right) & \rightarrow 1 \operatorname{sns}\left({ }^{1} S\right) \epsilon p\left({ }^{2} P\right) \\
& \rightarrow 1 s n s\left({ }^{3} S\right) \epsilon p\left({ }^{2} P\right) \\
& \rightarrow 1 \operatorname{snp}\left({ }^{1} P\right) \epsilon s\left({ }^{2} P\right) \\
& \rightarrow 1 \operatorname{snp}\left({ }^{3} P\right) \epsilon s\left({ }^{2} P\right)
\end{aligned}
$$

The $\mathrm{Li} 1 s$ main lines are represented by processes (1a) and (1b) for $n=2$. For $n>2$ the same events represent shakeup transitions, whereas the conjugate shakeup processes are described by events (1c) and (1d). Figure 1 shows a schematic description of these processes in terms of shake theory separating the transition matrix element in a dipole and monopole part, the latter representing basically the overlap matrix element between the relaxed and unrelaxed orbitals.

Due to the Pauli principle and spin conservation in the dipole excitation, triplet and singlet coupled states origi- shakeup

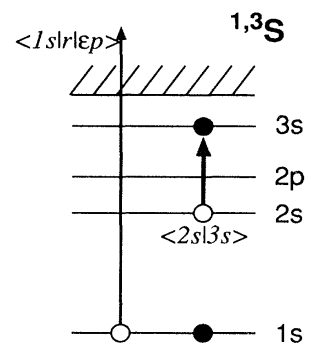

\section{conjugate shakeup}

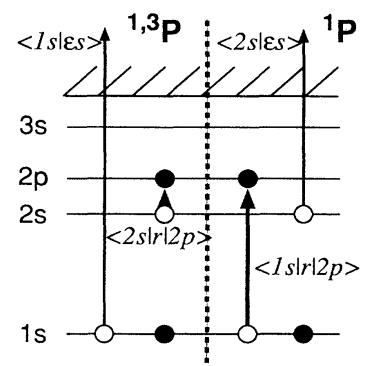

FIG. 1. Principal level scheme showing the excitation and ionization pathways of $\mathrm{Li} 1 s$ shakeup and conjugate shakeup processes. 
nate from different conjugate shakeup processes with the triplet state being populated only via a $\langle 1 s \mid \epsilon s\rangle$ monopole transition. Therefore there was considerable theoretical interest in a measurement where all components of the $\mathrm{Li}$ $1 s$ spectrum are clearly resolved. Up to now this requirement was only partially fulfilled; the interesting ${ }^{3} P$ conjugate shakeup component was barely resolved from the ${ }^{1} S$ main line. $9-11$

In this Rapid Communication we report on the $\mathrm{Li} 1 s$ photoelectron spectrometry experiment succeeding in resolving all spectral components and following their fractional intensity and angular-distribution asymmetry parameter from the sudden limit to the near-threshold region. The experiment was performed to test two predictions based on the conjugate shakeup model along with angular momentum transfer theory in the near-threshold region. (i) The satellite to the main-line branching ratio increases towards threshold because of increasing overlap between bound and continuum states near threshold. (ii) In the conjugate shakeup process the angular momentum of the photon is taken over by the excitation in the ionic core. Therefore, without interchannel coupling, the photoelectron carries no angular momentum, resulting in isotropic emission for a $1 s$ electron which corresponds to a $\beta$ value of zero. Assuming in the sudden limit a considerable fraction of configuration mixing, but assuming near threshold that most of the satellite intensity is due to conjugate shakeup and most of the higher $l$ contributions due to interchannel coupling being suppressed, ${ }^{12}$ one would expect decreasing $\beta$ behavior from 2 to 0 on approaching threshold from higher energies. Both qualitative predictions are well confirmed by the present data. Testing the capability of present theory to handle the many electron conjugate shakeup problem, we compare our results with the first $a b$ initio calculations based upon relaxed Hartree-Fock functions. ${ }^{13}$

The high-resolution Li experiment was performed at the Hamburger Synchrotronstrahlungslabor (HASYLAB) at DESY in Hamburg and in part at the Berliner Elektronenspeicherring für Synchrotronstrahlung (BES$\mathrm{SY}$ ) in Berlin by angle-resolved photoelectron spectroscopy using synchrotron radiation. Two time-of-flight spectrometers are used to measure partial cross sections, angular distribution, and asymmetry parameters simultaneously. A rotatable vacuum chamber allows the calibration of the degree of polarization of the incoming radiation and offers, in addition, determination of angular distributions more accurately by measuring time-of-flight spectra under various angles. A more complete description is given in former publications. ${ }^{14,15} \mathrm{~A}$ particular concern of this experiment besides the combined electron energy and photon energy resolution, was the control and stabilization of the atomic beam oven. We used a power controlled resistively heated oven with a $2-\mathrm{mm}$-diam nozzle heated slightly above crucible temperature $(970 \mathrm{~K})$ to avoid sticking of the nozzle. The high photon resolution necessary for the success of the experiment was achieved at the 5-m toroidal grating monochromator at HASYLAB using a new 1500 lines/mm grating, and at the new undulator beam line at BESSY also equipped with a toroidal grating monochromator. Both systems are described elsewhere. ${ }^{16,17}$ The

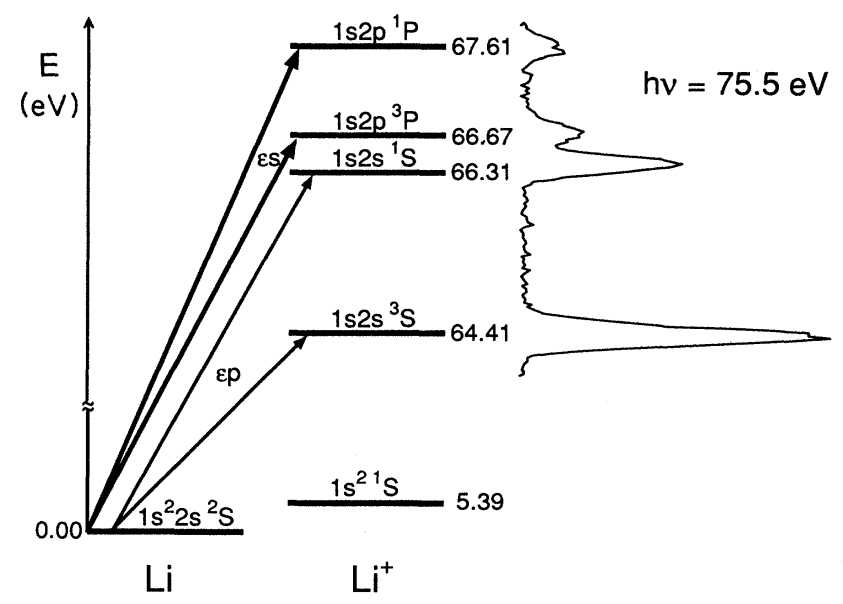

FIG. 2. Li $1 s$ photoelectron spectrum taken at $h v=75.5 \mathrm{eV}$ together with the corresponding energy level diagram.

challenge of all $\mathrm{Li} 1 s$ photoionization experiments was to resolve the ${ }^{1} S$ main-line component from the adjacent ${ }^{3} P$ component of the conjugate shakeup satellite. Figure 2 shows a $\mathrm{Li} 1 s$ energy-level diagram with proper relative energy spacings together with a photoelectron spectrum taken at photon energy of $75.5 \mathrm{eV}$. The ${ }^{1} S$ and ${ }^{3} P$ lines, separated by only $360 \mathrm{meV}$ from each other, are clearly resolved. A series of photoelectron spectra with comparable resolution were taken between 74- and 150-eV photon energy. Both analyzers were positioned in the $0^{\circ}$ and magic angle position sequentially allowing a cross check of the $\beta$ values obtained by the simultaneous two-analyzer method. The results of the experiment are displayed in Figs. 3 and 4.

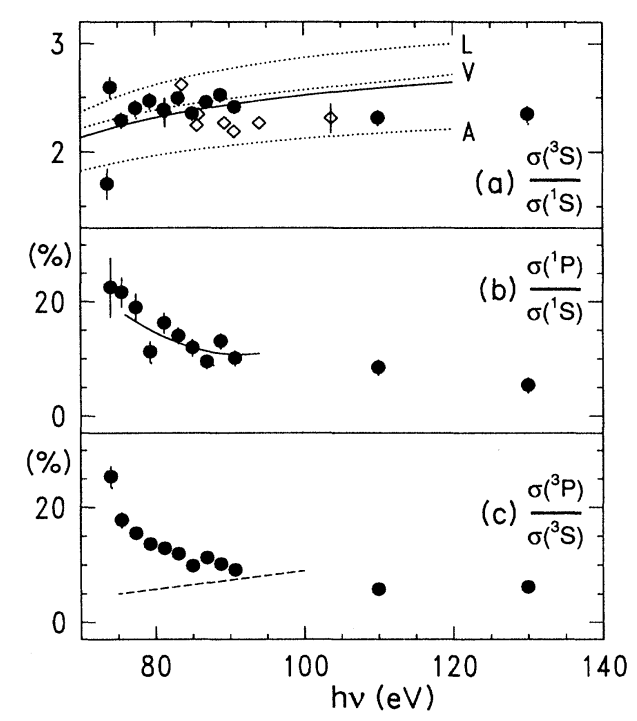

FIG. 3. Branching ratios of (a) main-line components $\sigma\left({ }^{3} S\right) / \sigma\left({ }^{\prime} S\right)$, (b) satellite to main-line $\sigma\left({ }^{1} P\right) / \sigma\left({ }^{\prime} S\right)$, and (c) $\sigma\left({ }^{3} P\right) / \sigma\left({ }^{3} S\right)$. The lozenges are from Ref. 9 as cited in Ref. 11 . The curves represent theoretical results by the relaxed HartreeFock (RHF) method from Ref. 13, as described in the text. 


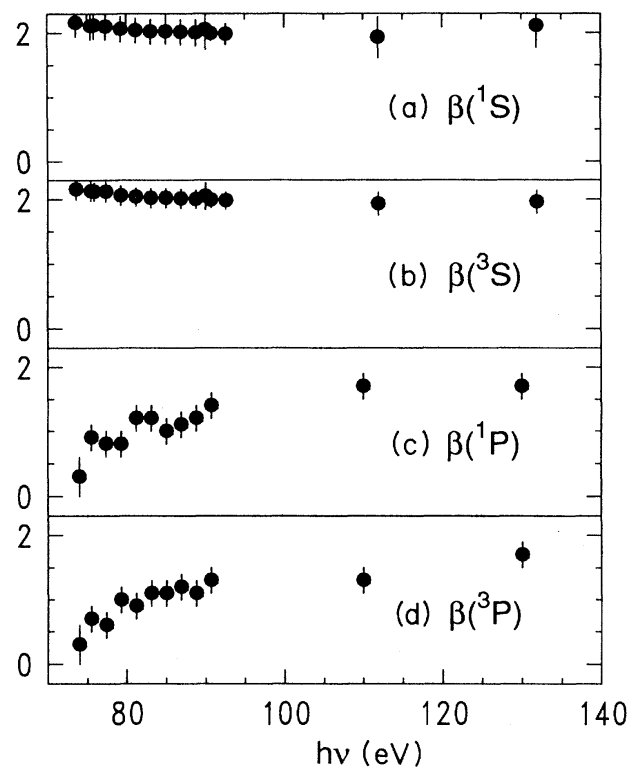

FIG. 4. Angular-distribution asymmetry parameter $\beta$ for the two main lines (a) ${ }^{1} S$ and (b) ${ }^{3} S$ and the two conjugate shakeup satellites (c) ${ }^{1} P$ and $(d){ }^{3} P$.

Figure 3 shows the branching ratio between the two main-line components ${ }^{3} S$ and ${ }^{1} S$ together with the satellite to main-line branching ratios $\sigma\left({ }^{1} P\right) / \sigma\left({ }^{1} S\right)$ and $\sigma\left({ }^{3} P\right) / \sigma\left({ }^{3} S\right)$ of the two conjugate shakeup transitions. Our $\sigma\left({ }^{3} S\right) / \sigma\left({ }^{1} S\right)$ branching ratio is in good agreement with the results of previous experiments ${ }^{9,11}$ if one corrects the results of Ref. 11 by our $\sigma\left({ }^{3} P\right) / \sigma\left({ }^{1} S\right)$ branching ratio. Considering the branching ratio between the two lines which were resolved in all investigations, $\sigma\left({ }^{1} P\right) / \sigma\left({ }^{3} S\right)$, there is also reasonable agreement between the different experiments. For the separated satellite to main-line branching ratios $\sigma\left({ }^{1} P\right) / \sigma\left({ }^{1} S\right)$ and $\sigma\left({ }^{3} P\right) / \sigma\left({ }^{3} S\right)$ there are no experimental data available for comparison, but they may be compared with theoretical $a b$ initio calculations by Armen et al. ${ }^{13}$ Because there is a relatively large scatter between the "length," "velocity," and "acceleration" results in these calculations, we compare our results essentially to the average of these three forms of the dipole matrix element rather than to the three forms independently, the more detailed comparison being applied only to the $\sigma\left({ }^{3} S\right) / \sigma\left({ }^{1} S\right)$ main-line branching ratio.

Considering the conjugate shakeup satellites there is very good agreement between theory and experiment for the $\sigma\left({ }^{1} P\right) / \sigma\left({ }^{1} S\right)$ satellite to main-line branching ratio. This shows that Hartree-Fock (HF) theory using relaxed orbitals is principally able to describe the conjugate shakeup process properly. However, special complications such as the existence of the Li-optical final state, a state supposed in first order not to contribute to the ionization cross section, may require treatment of continuum interactions beyond the scope of HF theory. This is illustrated by the even qualitatively poor agreement between theory and experiment in the case of the ${ }^{3} P$ conjugate shakeup state. This state, populated in the conjugate shakeup model only via $\langle 1 s \mid \epsilon s\rangle$ monopole ionization associated with a $\langle 2 s|r| 2 p\rangle$ dipole excitation, suffers from an orthogonality condition on the $1 s$ and $\epsilon S$ wave functions. This condition results from the orthogonality requirement imposed on the $1 s^{2}\left({ }^{1} S\right) 2 p\left({ }^{2} P\right)$ optical final state and the $1 s 2 s\left({ }^{1} S\right) \epsilon{ }^{2} P$ continuum state $\left\langle 1 s^{2}\left({ }^{1} S\right) 2 p{ }^{2} P \mid 1 s 2 s\left({ }^{1} S\right) \epsilon p{ }^{2} P\right\rangle$

$$
=\sqrt{2}\langle 1 s \mid 1 s\rangle\langle 1 s \mid 2 s\rangle\langle 2 p \mid \epsilon p\rangle=0
$$

giving no intensity to the ${ }^{3} P$ state in first order. Only if one takes final-state configuration mixing with the strong ${ }^{3} S$ state into account will the ${ }^{3} P$ state gain some intensity. The partial cross section resulting from this intensity borrowing process is displayed in Fig. 3(c) as a dashed line. Even this improved result is in poor agreement with the experimental data. This satellite line, therefore, still represents an important future challenge for the theoretical explanation of the $\mathrm{Li} 1 s$ conjugate shakeup problem; many-body perturbation theory or close-coupling calculations may be necessary to adequately describe continuum configuration interaction in the final state as in helium.

Figure 4 shows our results for the angular-distribution asymmetry parameter $\beta$. Here neither experimental nor theoretical data are available for comparison. However, conclusions drawn from simple application of angularmomentum transfer theory to the conjugate shakeup model as outlined above predict decreasing $\beta$ behavior from 2 to 0 by approaching threshold from higher energies. This behavior, also seen for photoionization of helium leaving the $\mathrm{He}^{+}$ion in a $2 p$ state, ${ }^{4}$ is strongly substantiated by our experimental data. Regarding the alignment of the remaining ion, an increasing alignment towards threshold is expected as already observed for helium $1 s$ and beryllium $1 s$ photoionization by Jiménez-Mier, Caldwell, and Ederer $^{18}$ and Krause and Caldwell. ${ }^{19}$ Considering both, the behavior of $\sigma$ and $\beta$, the particular disagreement observed for the fractional intensity of the $\mathrm{Li}^{3} P$ conjugate shakeup state seems to be of special character.

In summary, in contrast to previous investigations we have reported a high-resolution Li $1 s$ photoelectron spectrometry experiment separating all diagram and satellite components from each other. Our measurements provide both fractional intensities and angular-distribution asymmetry parameters of conjugate shakeup lines, allowing us to compare them unambiguously with different theoretical predictions particularly concerning their threshold behavior. Qualitative predictions on $\sigma$ and $\beta$ based on general arguments in this energy regime could be clearly confirmed. However, quantitative comparison with $a b$ initio calculations based on relaxed Hartree-Fock orbitals leads only to partially good agreement; a theoretical reevaluation of the $\mathrm{Li} K$-shell photoionization problem taking interchannel coupling adequately into account seems to be required.

The authors would like to thank Professor Sonntag and the members of his research group for their assistance and continuing interest. We also gratefully acknowledge very helpful discussions with Professor Larkins. One of us is indebted to the Deutsche Forschungsgemeinschaft for a research grant. This work was supported by the Bundesminister für Forschung und Technologie under Contract No. 05414CAB7. 
${ }^{1}$ M. Ya. Amusia, in Atomic Photoeffect, Physics of Atoms and Molecules, edited by P. G. Burke and H. Kleinpoppen (Plenum, New York, 1990).

${ }^{2}$ H. P. Kelly, in Proceedings of the Fifteenth International Conference on X-Ray and Inner-Shell Processes, Knoxville, $T N$, edited by M. O. Krause, S. T. Manson, and M. A. Carlson, AIP Conf. Proc. (AIP, New York, in press).

${ }^{3}$ U. Becker and D. A. Shirley, Phys. Scr. T31, 56 (1990).

${ }^{4}$ V. L. Jacobs and P. G. Burke, J. Phys. B 5, L67 (1972).

${ }^{5}$ J. M. Bizau, F. J. Wuilleumier, P. Dhez, D. L. Ederer, T. N. Chang, S. Krummacher, and V. Schmidt, Phys. Rev. Lett. 48, 588 (1982).

${ }^{6}$ F. J. Wuilleumier, Ann. Phys. (Paris) 4, 231 (1982).

${ }^{7}$ V. Schmidt, H. Derenbach, and R. Malutzki, J. Phys. B 15, L523 (1982).

${ }^{8}$ D. W. Lindle, T. A. Ferrett, U. Becker, P. H. Kobrin, C. M. Truesdale, H. G. Kerkhoff, and D. A. Shirley, Phys. Rev. A 31, 714 (1985).

${ }^{9} \mathrm{P}$. Gérard, Ph.D. Thèse, Université de Paris-Sud, 1984 (unpublished).

${ }^{10} \mathrm{P}$. Gérard, D. Cubaynes, J. M. Bizau, and F. J. Wuilleumier, J. Phys. (Paris) Colloq. 48, C9-719 (1987).

${ }^{11}$ T. A. Ferrett, D. W. Lindle, P. A. Heimann, W. D. Brewer, U. Becker, H. G. Kerkhoff, and D. A. Shirley, Phys. Rev. A 36, 3172 (1987).
${ }^{12}$ This supression may be explained within a one-electron picture due to centrifugal barrier effects [J. W. Cooper, Phys. Rev. Lett. 13, 762 (1964)]; but a more appropriate description regarding interchannel coupling would rely on interference effects between different dipole representation channels [P. C. Ojha, J. Phys. B 17, 1807 (1984)], rather than on the general influence of an effective potential.

${ }^{13}$ G. B. Armen, B. I. Craig, F. P. Larkins, and J. A. Richards, J. Electron. Spectrosc. Relat. Phenom. 51, 183 (1990); F. P. Larkins (private communication).

${ }^{14}$ U. Becker, Vacuum 38, 597 (1988).

${ }^{15}$ U. Becker, D. Szostak, H. G. Kerkhoff, M. Kupsch, B. Langer, R. Wehlitz, A. Yagishita, and T. Hayaishi, Phys. Rev. A 39, 3902 (1989).

${ }^{16}$ R. Bruhn, E. Schmidt, H. Schröder, B. Sonntag, A. Thevenon, G. Passereau, and J. Flamand, Nucl. Instrum. Methods Phys. Res. 208, 771 (1983).

${ }^{17}$ W. Peatman, C. Carbone, W. Gudat, and W. Heinen, P. Kuske, J. Pflügler, F. Schäfers, and T. Schroeter, Rev. Sci. Instrum. 60, 1445 (1989).

${ }^{18}$ J. Jiménez-Mier, C. D. Caldwell, and D. L. Ederer, Phys. Rev. Lett. 57, 2260 (1986).

${ }^{19}$ M. O. Krause and C. D. Caldwell, Phys. Rev. Lett. 59, 2736 (1987). 